\title{
Autotransplante cardíaco: um novo método no tratamento de problemas cardíacos complexos
}

Randas J. V. BATISTA*, Marcos FRANZONI*, Dalton PRÉCOMA*, Lise BOCHINO, Paulo NERY*, Eduardo OLIVEIRA*, Rosane CARVALHO*, Noriaki TAKESHITA*, Murilo FURUKAWA, Lisias THOME*', Francisco J. LINO**, José Luiz Verde dos SANTOS*, Marco A. CUNHA**

RBCCV 44205-264

BATISTA, R. J. V.; FRANZONI, M.; PRÉCOMA, D.; BOCHINO, L.; NERY, P.; OLIVEIRA, E.; CARVALHO, R.; TAKESHITA, N.; FURUKAWA, M.; THOME, L.; LINO, F. J.; SANTOS, J. L. V.; CUNHA, M. A. Autotransplante cardiaco: um novo método no tratamento de problemas cardíacos complexos. Rev. Bras. Cir. Cardiovasc., 10 (2): 90-100, 1995.

RESUMO: No período de janeiro de 1990 a maio de 1995 foram operados com a técnica do autotransplante cardiaco 92 pacientes com cardiopatias complexas e arritmias supraventriculares, principalmente fibrilação atrial $(n=89)$, reentrada $(n=2), Q T$ longo $(n=1)$. O sexo feminino predominou $(n=63)$. A idade variou de 18 a 76 anos $(m=43)$. Os defeitos concomitantes foram: átrio esquerdo gigante (medido pelo ecocardiograma $>6 \mathrm{~cm}$ ) $(n=65)$; átrio direito gigante $(n=9)$; átrio esquerdo aumentado $(<6 \mathrm{~cm} />4 \mathrm{~cm})(n=23)$; estenose mitral $(n=46)$; insuficiência mitral $(n=28)$; dupla lesão mitral $(n=16)$; estenose aórtica $(n=12)$; insuficiência aórtica $(n=5)$; insuficiência tricúspide $(n=78)$; trombose atrial $(n=23)$; calcificação atrial $(n=12)$; hipertensão pulmonar $(n=86)$; fibroelastose biventricular $(n=3)$; rotura atrioventricular (pós-troca de valva mitral) $(n=1)$; aneurisma da raiz aórtica $(n=1)$; ventriculectomia parcial $(n=8) ; 88$ pacientes saíram do centro cirúrgico em ritmo sinusal e assim permaneceram; 6 precisaram de drogas inotrópicas e 3 de drogas antiarrítmicas. Todos os pacientes que apresentavam átrio esquerdo ou direito gigante com fibrilação atrial tiveram seus átrios reduzidos ao tamanho normal. Não houve mortalidade operatória e 6 evoluíram a óbito hospitalar. Na reavaliação aos seis meses de pós-operatório, os sobreviventes estavam bem, em ritmo sinusal. A técnica do autotransplante cardíaco facilita o reparo intracardíaco, proporciona a redução atrial e conseqũente retorno do paciente ao ritmo sinusal e abre novas perspectivas.

DESCRITORES: Autotransplante cardiaco. Transplante cardiaco, humano, autotransplante.

\section{INTRODUÇÃO}

Pierre Simon, garoto prodígio nascido na Normandia, no século XVIII, tornou-se um físico brilhante. Pelos seus feitos, chegou a Marquês de Laplace (Figura 1). Na medicina, seus princípios são de fundamental importância, mais específicamente aplicados nas cirurgias do cólon e dos aneurismas da aorta 127.

A técnica do autotransplante cardíaco aplicada a pacientes já foi relatada por outros autores 26,145 , apesar de poucos casos, e de ser pouco difundida na literatura mundial. Ao que nos consta, depois de profunda pesquisa na literatura médica indexada, é a primeira vez que esta técnica é empregada no tratamento da fibrilação atrial.

O presente estudo visa demonstrar que o autotransplante cardíaco pode ser um importante auxílio tanto na correção anatômica de cardiopatias complexas, como na reversão das arritmias através da redução dos átrios. Com a redução para seus tamanhos normais, a diminuição da tensão da parede atrial (Figura 1B, C) pode ser suficiente para retornar os pacientes a ritmo sinusal e, conseqüentemente, melhorar seus débitos cardíacos.

\footnotetext{
Trabalho realizado no Centro Médico Caron-Curitiba, PR, e no IASERJ, Rio de Janeiro, RJ, Brasil.

Recebido para publicação em maio de 1995.

* Do Centro Médico Caron, Curitiba, PR.

** Do IASERJ. Rio de Janeiro, RJ.

Endereço para correspondência: Randas J. V. Batista. Fax: (041) 772-1523.
} 
BATISTA, R. J. V.; FRANZONI, M.; PRÉCOMA, D.; BOCHINO, L.; NERY, P.; OLIVEIRA, E.; CARVALHO, R.; TAKESHITA, N.; FURUKAWA, M.; THOME, L.; LINO, F. J.; SANTOS, J. L. V.; CUNHA, M. A. - Autotransplante cardíaco: um novo método no tratamento de problemas cardíacos complexos.. Rev. Bras. Cir. Cardiovasc., 10 (2): xx-xx, 1995.
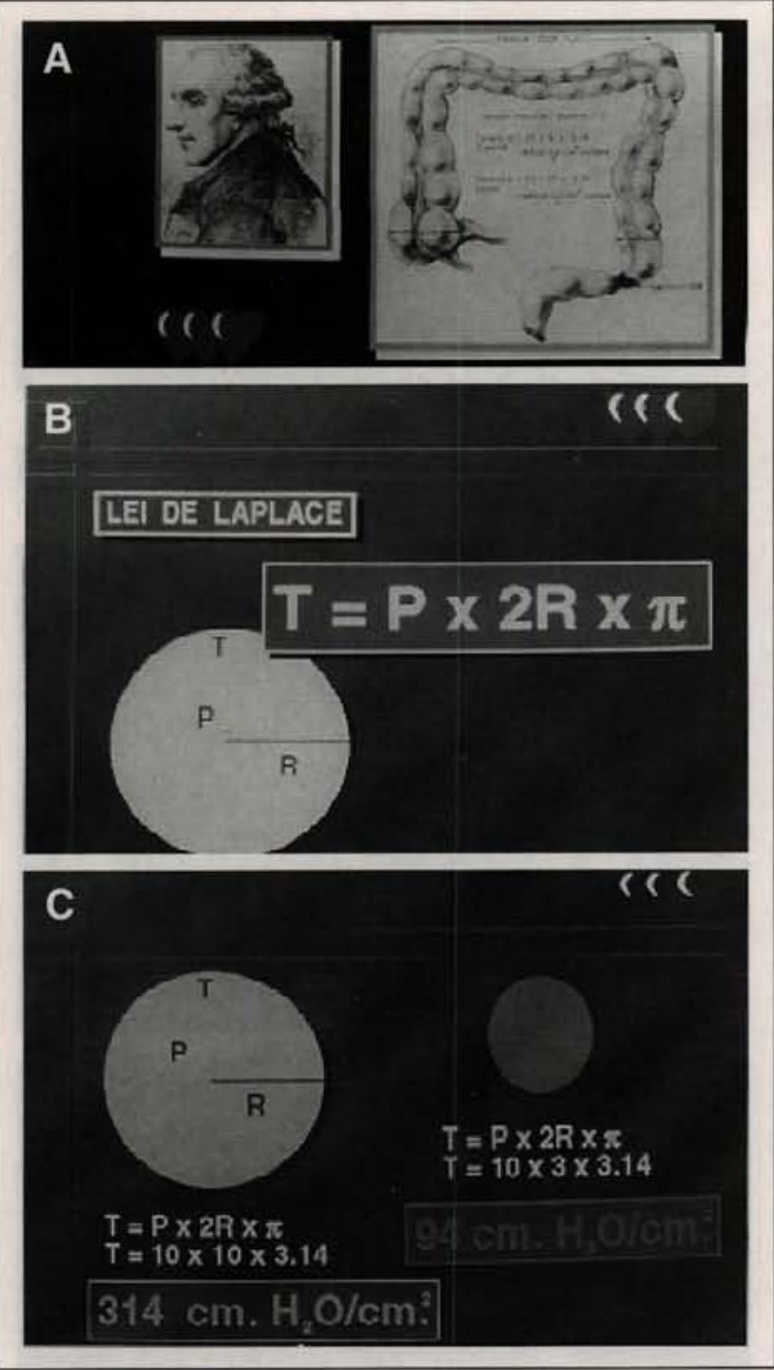

Figura 1

\section{MATERIAL E MÉTODOS}

De janeiro de 1990 até maio de 1995, foram realizadas 92 cirurgias cardiacas através da técnica do autotransplante cardíaco no Centro Médico Caron, em Campina Grande do Sul, região metropolitana de Curitiba, Paraná, e IASERJ, no Rio de Janeiro. A idade dos pacientes variou de 18 a 76 anos (média 43 anos). O sexo feminino predominou (63 pacientes).

A avaliação inicial foi realizada a nível ambulatorial, tendo sido analizados os parâmetros clínicos, eletrocardiográficos, radiológicos, ecocardiográficos e estudo hemodinâmico dos pacientes, assim obtendo os subsídios indispensáveis à programação cirúrgica.
Os defeitos encontrados, quando da avaliação e seleção dos casos, foram: átrio esquerdo gigante (assim considerado quando suas medidas ultrapassaram $6 \mathrm{~cm}$ pelo ecocardiograma), observado em $65(70,6 \%)$ pacientes; átrio direito gigante em 9 $(9,79 \%)$ pacientes; átrio esquerdo aumentado (quando menor que $6 \mathrm{~cm}$ e maior que $4 \mathrm{~cm}$ pelo ecocardiograma), em 23 (25\%) pacientes; estenose mitral em $46(50 \%)$ pacientes; insuficiência mitral em $28(30,44 \%)$ casos; dupla lesão mitral em 16 $(17,4 \%)$; estenose aórtica em $12(13,05 \%)$; insuficiência aórtica em $5(5,44 \%)$; insuficiência tricúspide em $78(84,79 \%)$; trombose atrial em $23(25 \%)$; hipertensão pulmonar em $86(93,48 \%)$; calcificação atrial 12 (13,05\%); fibroelastose biventricular em 2 $(2,18 \%)$; rotura atrioventricular (pós troca de valva mitral) em $1(1,09 \%)$; aneurisma da raiz aórtica em $1(1,09 \%)$ e $8(8,70 \%)$ casos de ventriculectomia parcial.

As arritmias supraventriculares consideradas foram, principalmente, fibrilação atrial observada em $89(96,74 \%)$ casos; arritmias por reentrada em 2 $(2,18 \%)$ casos; e $1(1,09 \%)$ caso de paciente sintomática com síndrome do QT longo.

\section{TÉCNICA CIRÚRGICA}

Com o paciente sob anestesia geral, o coração foi exposto através de uma esternotomia mediana. Após a heparinização $(3 \mathrm{mg} / \mathrm{kg})$, a aorta canulada no seu arco ao nível do tronco braquiocefálico ${ }^{108}$. A veia cava superior canulada na junção com as veias subclávias direita, inominada e jugular interna direita. A veia cava inferior canulada ao nível de sua junção com as veias supra-hepáticas. Após entrar em circulação extracorpórea, a aorta clampeada, iniciou-se a infusão de solução cardioplégica sangüínea normotérmica contínua, $200 \mathrm{ml} / \mathrm{min}$, na relação 4 de sangue: 1 de solução cristalóide (Tabela 1) pelo seio venoso (retroplegia). Mantivemos normotérmicas tanto a circulação sistêmica quanto a retroplegia. A veia cava superior foi incisada ao nível da veia grande ázigos (2 $\mathrm{cm}$ acima do nó sinusal) para não danificar o nó (Figura 2). Em

TABELA 1

SOLUÇĀO CARDIOPLÉGICA CRISTALÓIDE MISTURADA COM SANGUE NA RAZÃO DE 4:1

\begin{tabular}{lr} 
Soro glicosado & $500 \mathrm{ml}$ \\
Aspartato/Glutamato & $40 \mathrm{ml}$ \\
NaHCO3 & $10 \mathrm{ml}$ \\
MgSO4 & $10 \mathrm{ml}$ \\
KCL 19.1\% & $10 \mathrm{ml}$ \\
Manitol & $10 \mathrm{ml}$ \\
Insulina simples & $10 \mathrm{U}$ \\
Xilocaina 2\% & $2 \mathrm{ml}$ \\
Papaverina & $2 \mathrm{ml}$ \\
\hline
\end{tabular}


BATISTA, R. J. V.; FRANZONI, M.; PRÉCOMA, D.; BOCHINO, L.; NERY, P.; OLIVEIRA, E.; CARVALHO, R.; TAKESHITA, N.; FURUKAWA, M.; THOME, L.; LINO, F. J.; SANTOS, J. L. V.; CUNHA, M. A. - Autotransplante cardíaco: um novo método no tratamento de problemas cardíacos complexos.. Rev. Bras. Cir. Cardiovasc., 10 (2): 90-100, 1995.

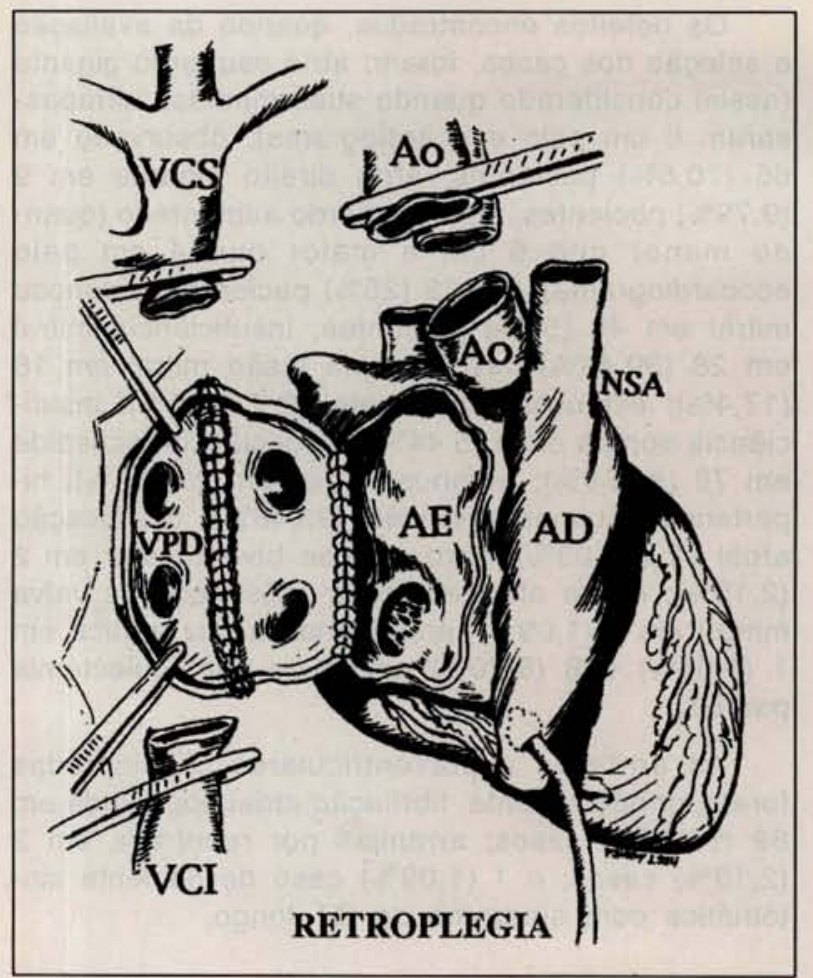

Figura 2

seguida, o átrio esquerdo foi incisado ao nível da veia pulmonar superior direita e esta incisão extendida em direção da aorta. A aorta foi incisada $3 \mathrm{~cm}$ acima da coronária direita e a artéria pulmonar incisada também no mesmo nível da aorta. Em seguida, esta incisão seguiu em direção da veia cava inferior, passando por baixo desta em direção à veia pulmonar inferior esquerda, onde se completa cincunferencialmente a incisão do átrio esquerdo. Por último, a incisão da veia cava inferior. Completados estes cortes, o coração ficou livre para sua exérese. $O$ coração foi avaliado em uma pequena cuba (Figura $3 \mathrm{~A}$ ), os trombos, quando presentes, retirados (Figura 3B, c). Eventual lesão associada (prótese mitral calcificada) foi corrigida sem nenhuma dificuldade (Figura 4A, B, C), uma vez que o coração estava numa cuba. Em seguida, foi feita a redução dos átrios para seus tamanhos normais. A aurícula esquerda foi suturada por dentro com um fio $3 \# 0$ de monofilamento de Polipropileno, como também o foi a fossa ovalis. A distância entre as veias pulmonares direitas e esquerdas diminuída com uma sutura contínua de $2 \# 0$ de Polipropileno (Figura 2). Depois de corrigir eventual lesão associada, o coração foi reimplantado com fio de Polipropileno $2 \# 0$. A anastomose se iniciou pelo átrio esquerdo em sua porção mais distal, a veia pulmonar inferior esquerda (Figura 2) terminando ao nível das veias pulmonares direitas. A veia cava inferior em seguida

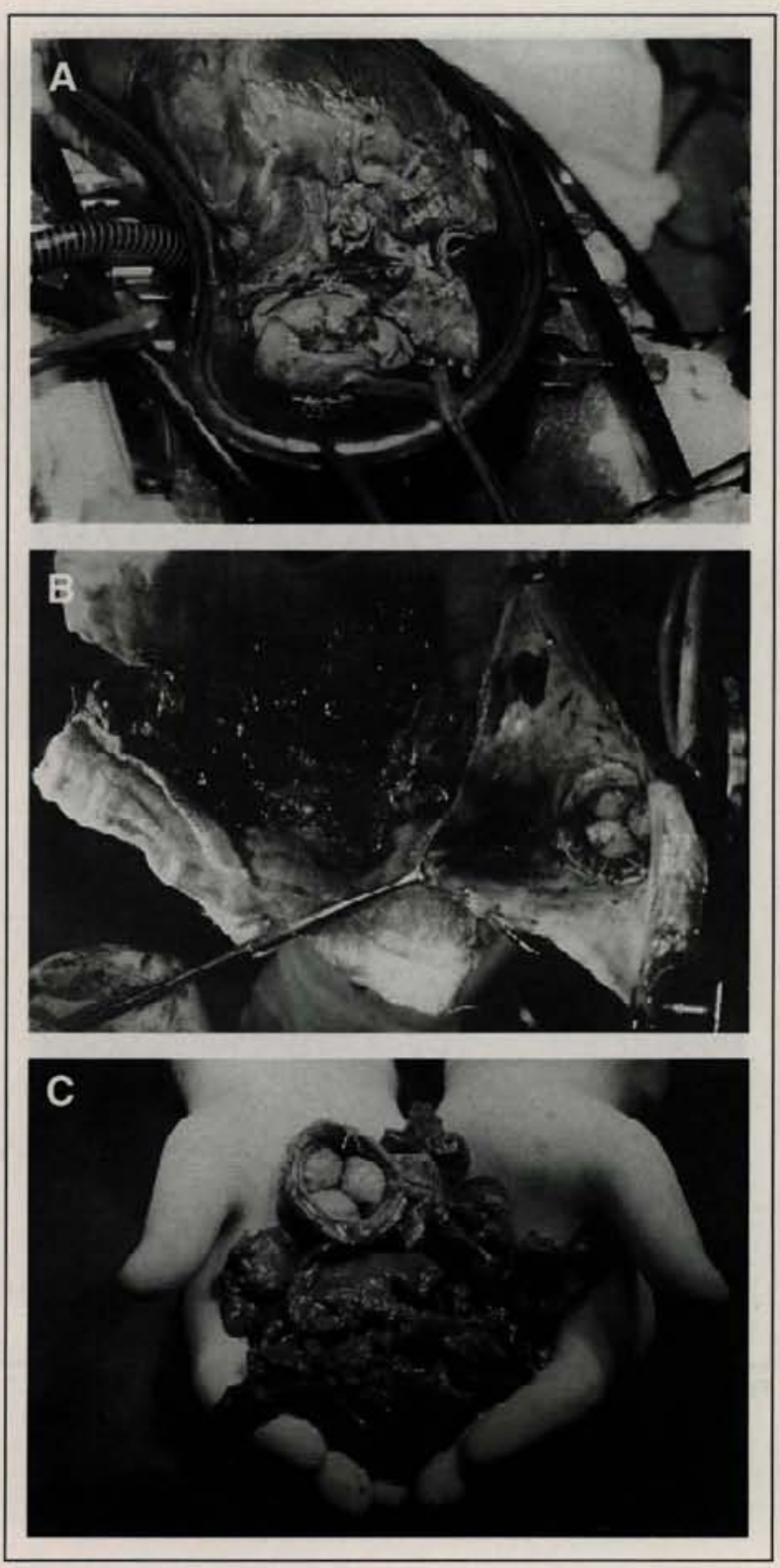

Figura 3

reanastomosada, seguida pela artéria pulmonar, aorta e por último a veia cava superior com fio $4 \# 0$ de Polipropileno. Todas as anastomoses foram feitas com sutura contínua simples e durante todo este tempo o coração foi mantido com um fluxo de 200 $\mathrm{ml} / \mathrm{min}$ (Figura 5) 29, 33. Ao desclampear a aorta, o coração adquiriu seu ritmo sinusal. 0 tempo de clampeamento aórtico variou de $40 \mathrm{~min}$ a $90 \mathrm{~min}$ (média de $58 \mathrm{~min}$ com desvio padrão de $+/-10 \mathrm{~min}$ ) e o de circulação extracorpórea variou entre $70 \mathrm{~min}$ a $130 \mathrm{~min}$ (média de $90 \mathrm{~min}$ com desvio padrão de +/- $13 \mathrm{~min}$ ). 
BATISTA, R. J. V.; FRANZONI, M.; PRÉCOMA, D.; BOCHINO, L.; NERY, P.; OLIVEIRA, E.; CARVALHO, R.; TAKESHITA, N.; FURUKAWA, M.; THOME, L.; LINO, F. J.; SANTOS, J. L. V.; CUNHA, M. A. - Autotransplante cardiaco: um novo método no tratamento de problemas cardiacos complexos..

Rev. Bras. Cir. Cardiovasc., 10 (2): xx-xx, 1995.
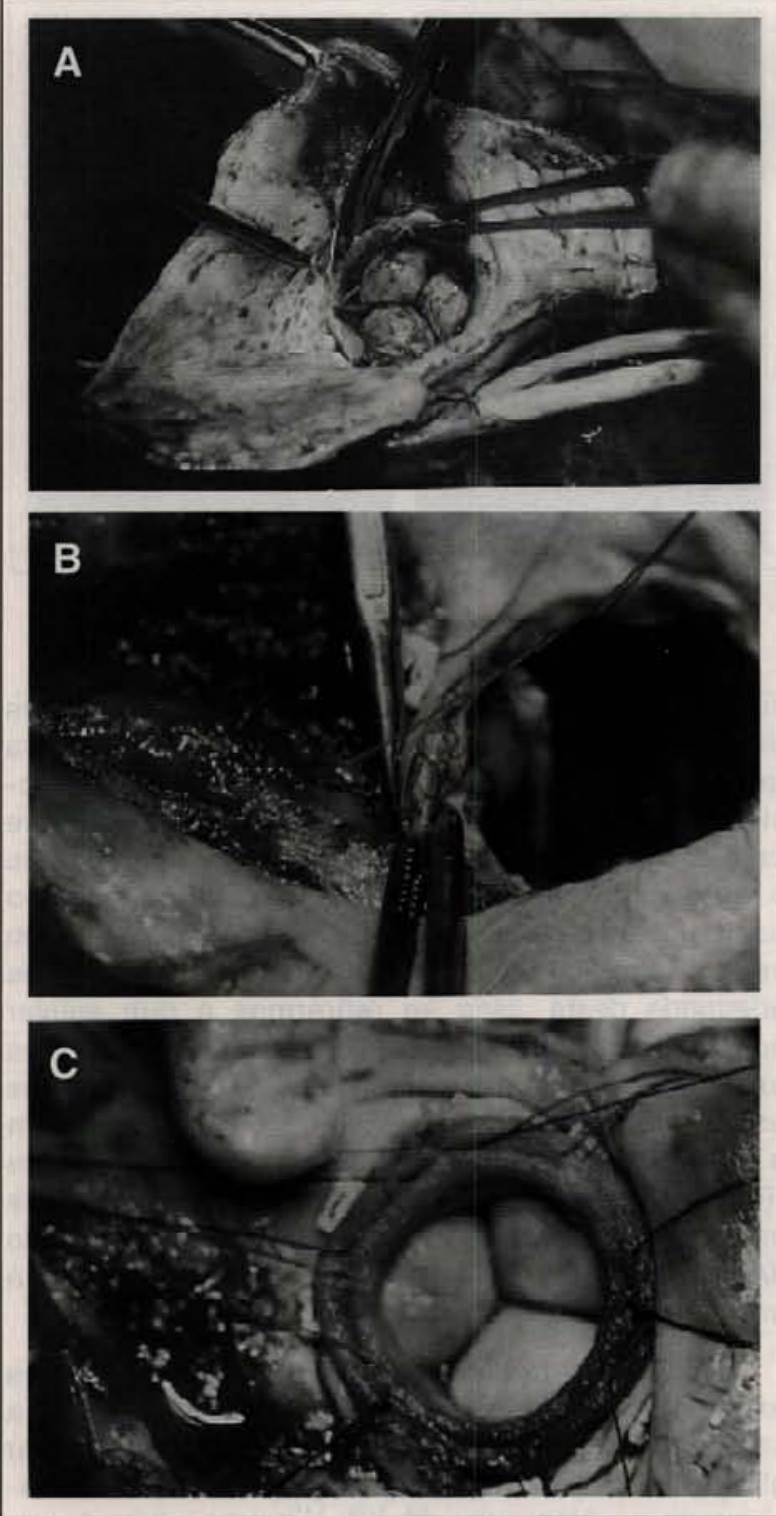

Figura 4

\section{RESULTADOS}

Oitenta e seis dos 92 pacientes operados (100\%) saíram do centro cirúrgico em ritmo sinusal, sem drogas inotrópicas ou antiarrítmicas e usamos, em todos, nitroprussiato. Foram avaliados de rotina por eletrocardiograma de 12 canais, estudo eletrocardiográfico dinâmico (sistema Holter Spacelabs, 2 canais), radiologia e ecodopplercardiografia colorida no pré-operatório imediato (Figuras 6 e 7 , respectivamente) e de seis em seis meses ambulatorialmente, com os mesmos exames. Todos os pacien-

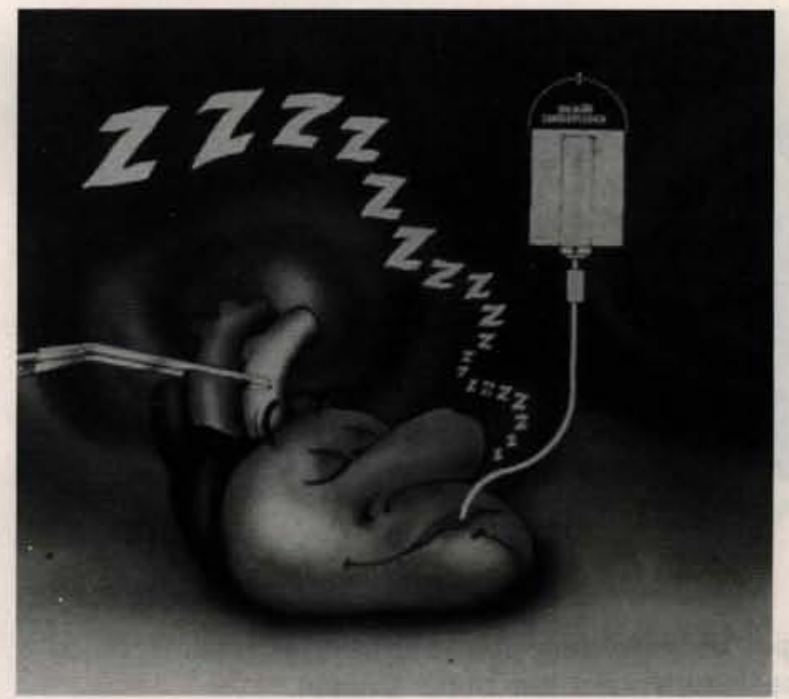

Figura 5

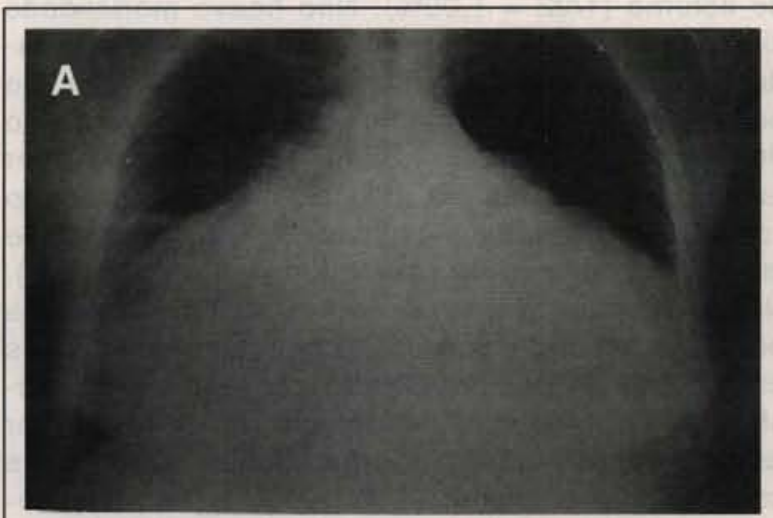

B

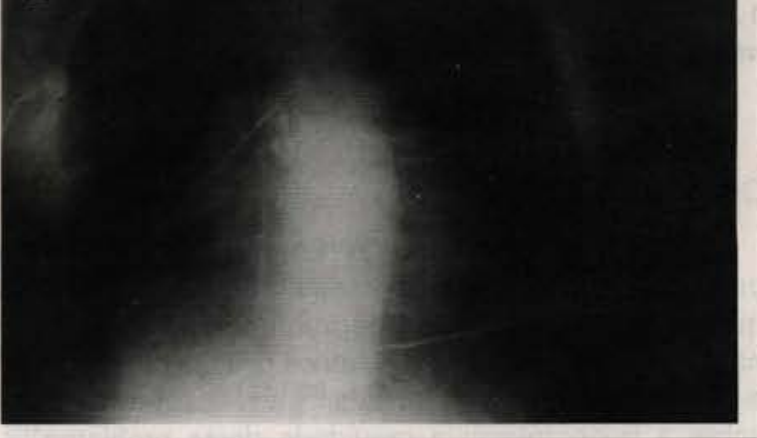

Figura 6

tes que apresentavam átrio esquerdo ou direito gigante com fibrilação atrial tiveram seus átrios reduzidos ao tamanho normal, conforme medidas ecocardiográficas realizadas no pós-operatório (Figura 7B). Na reavaliação após seis meses da cirur- 
BATISTA, R. J. V.; FRANZONI, M.; PRÉCOMA, D.; BOCHINO, L.; NERY, P.; OLIVEIRA, E.; CARVALHO, R.; TAKESHITA, N.; FURUKAWA, M.; THOME, L.; LINO, F. J.; SANTOS, J. L. V.; CUNHA, M. A. - Autotransplante cardiaco: um novo método no tratamento de problemas cardíacos complexos.. Rev. Bras. Cir. Cardiovasc., 10 (2): 90-100, 1995.

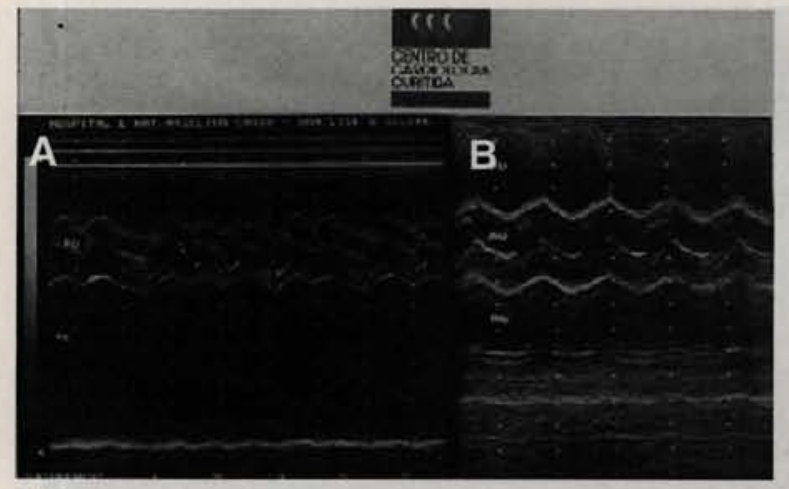

Figura 7

gia, os pacientes estavam bem ao exame físico e se mantiveram em ritmo sinusal.

A morbidade mais comum foi o sangramento (9/ $92=9,72 \%$ ), seguido de insuficiência respiratória $(6 / 92=6,63 \%)$, infecção $(3 / 92=3,26 \%)$, deiscência de esterno $(1 / 92=1,09 \%)$. Não houve mortalidade cirúrgica (ocorrida no centro cirúrgico). A mortalidade hospitalar (ocorrida nos primeiros 30 dias de pós-operatório) mais comum foi também distúrbio da coagulação $(4 / 92=4,35 \%)$; 1 óbito ocorreu por extubação precoce na UTI e o plantonista não conseguiu reentubar o paciente $(1 / 92=1,09 \%)$, e outro por trombose de cava inferior $(1 / 92=1,09 \%)$. Neste paciente sua veia cava inferior foi canulada pela veia femoral direita. A mortalidade tardia (mais de 30 dias de pós-operatório) ocorreu em um senhor de 75 anos com pneumonia de lobo superior esquerdo $(1 / 86=1,17 \%)$; uma senhora de 67 anos com embolia pulmonar $(1 / 86=1,17 \%)$ e outra senhora que foi admitida em outro hospital com sintomas de insuficiência cardíaca direita (sic) $(1 / 86=$ $1,17 \%) ; 42(45,66 \%)$ pacientes foram operados pela segunda vez, e $1(1,09 \%)$ pela terceira vez.

\section{COMENTÁRIOS}

Desde 1901, quando OWEN \& FENTON ${ }^{100}$ descreveram este extremo crescimento do átrio esquerdo, inúmeras outras comunicações apareceram na literatura 35,72 , inclusive de átrios direitos gigantes ${ }^{1,139}$ e até de gigantismos biatriais ${ }^{89,142}$. Discute-se muito sobre a fisiopatologia e gravidade deste problema ${ }^{103}$. Vários autores tentaram ressecção ${ }^{82}$, plicatura $66,67,71$, mas nenhum destes métodos são aceitos ainda como o tratamento ideal. Outros descrevem a plicatura com bons resultados pós-operatórios, mas seus pacientes continuaram em fibrilação atrial. Alegaram que estes bons resultados se deveram ao alívio destes átrios gigantes corrigidos nas estruturas vizinhas (brônquios pulmonares, ventrículo esquerdo,

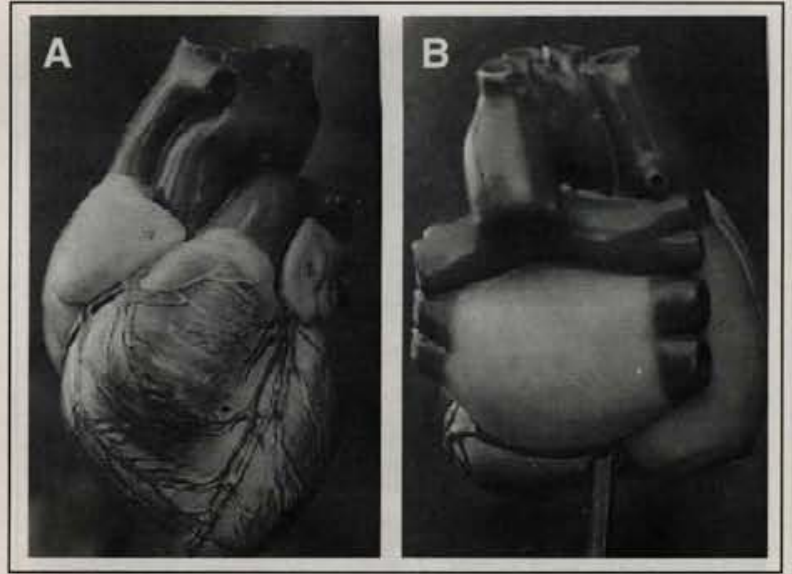

Figura 8

coronárias etc). Mas a grande maioria dos relatos da literatura consiste em descrever o fato, ora radiográfico, tomográfico ou simplesmente anátomoclínico. Nós começamos com o autotransplante cardíaco para corrigir esta lesão complexa porque o átrio esquerdo é, na realidade, inferior com o paciente em posição supina (Figura $8 \mathrm{~A}, \mathrm{~B}$ ). Assim sendo, acreditamos conseguir uma diminuição mais adequada deste átrio se retirarmos o que estiver acima (o coração). Não acreditamos que estes pacientes retornem ao ritmo sinusal se aplicarmos as técnicas de outros autores $28,34,55,60,65,150$ sem a redução do átrio (Figura $1 \mathrm{~B}, \mathrm{C}$ ) e neste paciente (Figura 9), o cálcio que aparece na radiografia precisamos de um pequeno martelo para retirá-lo da parede atrial. Já aparece na literatura brasileira um reflexo de nosso trabalho.

Nos casos de síndrome do QT longo, nós temos especial cuidado com o nó sinusal. Incisamos a cava superior $2 \mathrm{~cm}$ acima do nó sinusal para evitar bloqueio atrioventricular. A paciente autotransplantada na Alemanha 48, 109, 110 necessitou de um marcapasso "dual chamber" porque a incisão foi no

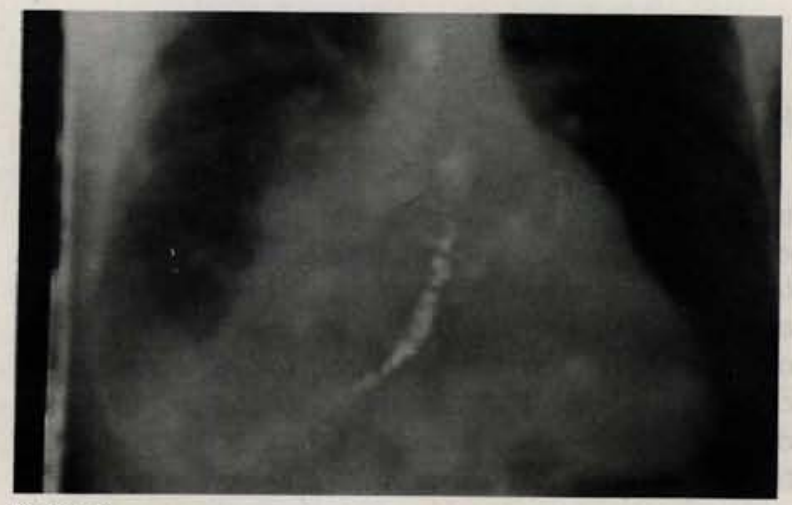

Figura 9 
BATISTA, R. J. V.; FRANZONI, M.; PRÉCOMA, D.; BOCHINO, L.; NERY, P.; OLIVEIRA, E.; CARVALHO, R.; TAKESHITA, N.; FURUKAWA, M.; THOME, L.; LINO, F. J.; SANTOS, J. L. V.; CUNHA, M. A. - Autotransplante cardíaco: um novo método no tratamento de problemas cardíacos complexos.. Rev. Bras. Cir. Cardiovasc., 10 (2): xx-xx, 1995.

átrio direito. A nossa paciente saiu em ritmo sinusal porque evitamos incisar o átrio direito. No InCór de São Paulo, recentemente, começaram também a fazer este tipo de cirurgia. Muito se discute ainda sobre síndrome do QT longo $10,43,64,109,110,145$.

Quanto ao autotransplante em si, é uma técnica consagrada graças aos estudos dos milhares de heterotransplantes já existentes e também de estudos dos próprios autotransplantes. Os pacientes têm uma boa recuperação, talvez graças aos novos métodos de proteção miocárdica 13,122 . Às vezes surge, no pós-operatório imediato, uma necessidade de aumentar a freqüência cardíaca do paciente. Devemos nos lembrar que eles não mais respondem à atropina ${ }^{18}$. Com o tempo, o teste ergométrico fica normal (resposta adrenérgica).

Pesquisamos exaustivamente na literatura médica indexada e não encontramos nenhum trabalho que se refira ao tratamento da fibrilação atrial com este método. Já tentamos todos os tipos de redução do átrio esquerdo por meio de plicaturas variadas, mas consideramos os resultados muito reservados. Pelo princípio de Laplace, a tensão do átrio esquerdo somente será igual à do átrio direito quando suas pressões e diâmetros forem iguais. Acreditamos que, para que o paciente retorne ao ritmo sinusal, a tensão da parede do átrio esquerdo deverá ser igual à do átrio direito. Nestas circuns- tâncias, o melhor e mais eficaz método para reduzir o átrio esquerdo é o autotransplante cardíaco.

\section{CONCLUSÃO}

A cirurgia cardíaca, em constante evolução, requer uma total interação entre os dados fornecidos pela clínica, os avanços tecnológicos progressivos e o desenvolvimento de novas técnicas cirúrgicas, na incessante procura de resultados cada vez mais positivos no tocante ao seu principal objetivo, que é a recuperação do paciente para uma vida normal, ou o mais próximo possível da normalidade. Assim sendo, o trabalho já é iniciado no préoperatório, através da seleção de rigorosos critérios para uma escolha precisa da técnica cirúrgica a ser adotada para cada caso. O desenvolvimento da técnica do Autotransplante Cardíaco vem somarse ao arsenal de recursos modernamente disponíveis ao cirurgião cardiovascular, facilitando o reparo intracardíaco e a redução atrial. Através desta, ocorre a redução da tensão da parede do átrio, o que, segundo a experiência deste Serviço, promove o retorno do coração do paciente ao ritmo sinusal, melhorando o débito cardíaco, possibilitando ao enfermo melhor qualidade de vida, com necessidade de menos medicamentos após a cirurgia.

BATISTA, R.J.V.; FRANZONI, M.; PRÉCOMA, D.; BOCHINO, L.; NERY,P.; OLIVEIRA, E.; CARVALHO, R.; TAKESHITA, N.; FURUKAWA, M.; THOMÉ, L.; LINO, F. J.; SANTOS, J. L. V.; CUNHA, M. A. - Heart autotransplantation: a new technique to complex intracardiac reppairs. Rev. Bras. Cir. Cardiovasc., 10 (2): 90-100, 1995.

ABSTRACT: From January 1990 to May 1995, 92 patients with complex cardiac problems and supraventricular arrhythmias were operated upon with the technique of heart autotransplantation. The arrhythmias were: atrial fibrillation ( $n=89)$; reentry $(n=2)$; long QT syndrome $(n=1)$. Females predominated $(n=63)$. The age varied from 18 to 76 years $(m=43)$. Concomittant defects were: giant left atrium ( $>6 \mathrm{~cm}$ measured by echo) $(n=65)$; giant right atrium $(n=9)$; large left atrium $(<6 \mathrm{~cm} . />4 \mathrm{~cm})(n=23)$; mitral stenosis $(n=46)$; mitral insufficiency $(n=28)$; mitral double lesion $(n=16)$; aortic stenosis $(n=12)$; aortic insufficiency $(n=5)$; tricuspid insufficiency $(n=75)$; atrial thrombosis $(n=23)$; atrial calcification $(n=12)$; pulmonnary hypertension $(n=86)$; biventricular fibroelastose $(n=3)$; atrioventricular rupture $(n=1)$; aortic root aneurysm $(n=1)$; partial ventriculectomy $(n=8) ; 88$ patients left the operating room and remained in sinus rhythm; 6 required inotropic drugs and 3 antiarrhythmic drugs. All patients with giant atria and atrial fibrillation had their atria reduced to normal sizes. There were no OR mortality and 6 patients died during hospitalization. Six months later the survivors were clinically well, in sinus rhythm. The technique of heart autotransplantation facilitates intracardiac reppairs, provides atrial reduction and returns patients with atrial fibrillation into sinus rhythm, and opens new frontiers.

DESCRIPTORS: Heart autotransplantation. Heart transplantation, human, autotransplantation. 
BATISTA, R. J. V.; FRANZONI, M.; PRÉCOMA, D.; BOCHINO, L.; NERY, P.; OLIVEIRA, E.; CARVALHO, R.; TAKESHITA, N.; FURUKAWA, M.; THOME, L.; LINO, F. J.; SANTOS, J. L. V.; CUNHA, M. A. - Autotransplante cardiaco: um novo método no tratamento de problemas cardiacos complexos.. Rev. Bras. Cir. Cardiovasc., 10 (2): 90-100, 1995.

\section{REFERÊNCIAS BIBLIOGRÁFICAS}

1 ASAYAMA, J.; MATSURA, T.; ENDO, N.; MATSUKUBO, H.; FURUKAWA, K. - Idiopathic enlargement of the right atrium. Am. J. Cardiol., 40: 620-623, 1977.

2 ASHWORTH, H. \& JONES, A. M. - Aneurysmal dilatation of the left auricle with erosion of the spine. Brit. Heart J., 8: 207-211, 1946.

3 BACH, F. \& KEITH, T. S. - Enlargement of the left auricle of the heart. Lancet, 2: 766, 1929.

4 BEDFORD, D. E.; Extreme dilatation of the auricle to the right. Am. Heart J., 3: 127, 1927.

5 BEDFORD, D. E. - Extreme dilatation of the left auricle to the right. American Heart Joumal, 3: 127-138, 1927.

6 BEHRENDT, D. N. \& ABERDEEN, E. - Congential aneurysm of the left atrium. Ann. Thorac. Surg., 13: 54-59, 1972.

7 BEPPU, S.; KAWAZOE, K.; NIMURA.; NAGATA, S.; PARK, Y.; SAKAKIBARA, H.; FUGITA,T. Echocardiographic study of abnormal position and motion of posterobasal wall of the left ventricle in case of giant left atrium. Am. J. Cardiol., 49: 467, 1982.

8 BERTRAND, M. E.; LABLANCHE, J. M.; TILMANT, P. Y.; DUCLOUX, G.; WAREN BOURG. H.; SOOTS, G. - Complete denervation of the heart (autotransplantation) for treatment of severe refractory coronary spasm. Am. J. Cardiol., 47: 1375, 1981.

BEXTON, R. S.; NATHAN, A. W.; HELLESTRAND, K. J. - Sinoatrial function after cardiac transplantation. J. Am. Coll. Cardiol.; 3: 712, 1984.

10 BHANDARI, A. K.; SCHEINMAN, M. M.; MORADY, F.; SVINARICH, J.; MASON, J., WINKLE, R. - Efficacy of left cardiac sympathectomy in the treatment of patients with the long QT syndrome. Circulation, 70: 1018-1023, 1984.

11 BISHOP, L. F. \& BABEY, A. - Massive left auricle. JAMA., 106: $462,1936$.

12 BORDET, E. - Les anomalies de développment du profil de l' oreillette gauche dans le rétrécissement mitral. J. Méd. Paris, 40: 227-229, 1922.

BRAILLE, D. M. - Como eu faço: Cardioplegia sangūinea isotérmica retrógrada de baixo volume. Rev. Bras. Cir. Cardiovasc., 7: 221-229, 1992.

14 BREDIKIS, I.; PUTELIS, R. A.; NORKUS, T. A. - Experimental use of the mechanical suture in autotransplantation of the heart. Kardiologiia, 8: 138140,1968

BROMWELL, J. C. \& DUGUID, J. B. - Aneurysmal dilatation of the left auricle. Quart. J. Med., 21: 187, 1927.

BURKHARDT, E. A. - Marked dilatation of the left auricle of the heart. Am. J. Pathol., 6: 463, 1930.

CACHERA, J. P.; LA COMBE, B.; SALAMAGNE, J. C.; BAUMANN, D.; DE LA FUENTE, A.; DUBOST, C. Orthotopic transplantation of the heart in dogs. Ann. Chir. Thorac. Cardiovasc., 5: 233-242, 1966.

Eletrophysiologic studies in the denervated transplanted human heart: Response to atrial pacing and atropine. Circulation Res., 32: 268, 1973.

19

20

21

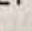

23

\section{COOL} ANGELINI, P. - Human cardiac explantation and autotransplantation: aplication in a patient with a large cardiac pheochromo cytoma. Texas Heart Inst. J., 12: $171-176,1985$.

27 CORELL, L. R. - Technics of pharmachological studies of coronary circulation: angiocardiocadiography and possibilities of fluorescent-coronary angiography and the orthotopic autotransplantation of the heart. Arch. Inst. Farmacol. Exp. (Madrid), 22: 63-100, 1970.

COX, J. L.; SCHUESSLER, R. B.; DAGOSTINO, H. J.; STONE, C. M.; CHANG, B. C.; CAIN, M. E.; CORR, P. B.; BOINEAU, J. P. - The surgical treatment of atrial fibrillation. J. Thorac. Cardiovasc. Surg., 101: 569-592, 1991.

DAGGETT, W. M.; SHANAHAN, E. A.; LEAK, L. V.; WARSHAW, J. B.; LOWER, R. R.; AUSTEN, W. G. - Functional, metabolic and structural changes in the chronic canine cardiac autotransplant. Bull. Soc. Int. Chir., 32: 223-228, 1973.

DAGGETT, W. M.; WILLMAN, V. L.; COOPER, T.; HANLON, C. R. - Work capacity and efficiency of the autotransplanted heart. Circulation, 45: 196-204, 1961.

DAGGETT, W. M.; WILLMAN, V. L.; HARADA, Y.; BARNER, H. B.; COOPER, T.; HANLON, C. R. Work capacity and efficiency of the autotransplanted heart. Surg. Forum, 17: 222, 1966.

DALEY, R. \& FRANKS, R. - Massive dilatation of the left auricle. Quart. J. Med., 18: 81, 1949.

33 DAWKINS, K. D.; HAVERISH, A.; DERBY, G. C.; SCOTT, W. C.; REITZ, B. A.; STINSON, E. B.; 
BATISTA, R. J. V.; FRANZONI, M.; PRÉCOMA, D.; BOCHINO, L.; NERY, P.; OLIVEIRA, E.; CARVALHO, R.; TAKESHITA, N.; FURUKAWA, M.; THOME, L.; LINO, F. J.; SANTOS, J. L. V.; CUNHA, M. A. - Autotransplante cardiaco: um novo método no tratamento de problemas cardíacos complexos.. Rev. Bras. Cir. Cardiovasc., 10 (2): 90-100, 1995.

JAMIESON, S. W.; SHUMWAY, N. E. - Long term hemodynamics following combined heart and lung transplantation in primates. J. Thorac. Cardiovasc. Surg., 89: 55-62, 1985.

34 DEFAUW, J. J.; VAN HEMEL, N.; VERMEULEN, F. Short term results of the "Corridor Operation". Circulation, II 43: 0172, (Resumo).

35 DE SANCTIS, R. W.; DEAN, D. C.; BLAND, F. E. - Extreme left atrial enlargement. Circulation, 29: 14-23, 1964.

36 DIALER, L. - Unusual enlargement of the left auricle, Ztschr. Kreislaufforsch., 30: 857, 1938.

DI EUSANIO, G.; GREGORINI, R.; MAZZOLA, A.; CLEMENTI, G.; POCACCINI, B.; CAVARRA, F.; TARASCHI, F.; ESPOSITO, G.; DI NARDO, W.; DI LUZIO, V. - Giant left atrium and mitral valve replacement: risk factor analysis. Eur. J. Cardiothorac. Surg., 2: 151-159, 1988.

DINES, D. E. \& ANDERSON, M. W. - Giant left atrium as a cause of dysphagia. Ann. Intern. Med., 65: 758761,1966

DISESA, V. J. \& COHN, L. H. - Experimental orthotopic heart autotransplantation. J. Heart Transplant., 7: 471473, 1988.

DONALD, D. E. - Capacity for exercise after denervation of the heart. Circulation, 38: 225, 1968.

41 DONALD, D. E. \& SHEPHERD, J. T. - Function of the dennervated heart. G. Clin. Med., 51: 1-7, 1970.

DONG, E.; HURLEY, D. G.; LOWER, R. R.; SHUMWAY, N. E. - Performance of the heart two years after autotransplantation. Surgery, 56: 270, 1964.

43 DRAKE, H. A. J.; CUMMINS, P.; ENGLISH, T. A. Metabolic changes in the autotransplanted baboon heart. Transplantation, 38: 454, 1984.

44 DUMONT, L.; STANLEY, P.; CHARTRAND, C. Hemodynamic effects of hypertonic sodium bicarbonate on denervated canine hearts. J. Heart Transplant, 4: 247-250, 1985.

45 EBERT, P. A. \& SABISTON, D. C. - Pharmacologic quantitation of cardiac sympathetic reinnervation. Surgery, 68: 123-127, 1970.

46 EMANUEL, J. G. - Extreme dilatation of the left auricle. Lancet, 1: 591, 1923.

47 EVAT, C. F. T. - Great dilatation of the left auricle. Lancet, 1: 1194, 1926.

48 FIEHRING, H.; WARNKE, H.; PECH, H. J.; MORGAN, P.; PFEIFFER, D. - Treatment of the long QT syndrome with heart transplantation: a case report. Eur. Heart J., 9: 46, 1988.

49 FRAMBACH, P. G.; GESKES. G. G; CHERIEX, E. C.; WELLENS, H. J.; PENN, D. C. - Giant intrapericardial aneurysm of the atrial appendage. Eur. Heart J., 11: 848-853, 1990.

50 FUGITA, T.; KAWAZOE, K.; BEPPU, S.; MANABE, $H$. - Surgical treatment of mitral valvular disease with giant left atrium. Jpn. Circ. J., 46: 420-426, 1982.
51 GODWIN, T. F.; AUGER, P.; KEY, T. A. - Intrapericardial aneurysmal dilation of the left atrial appendage. Circulation, 37: 397-401, 1968.

GOULD, L. A.; BETZU, R.; YANG, D. C.; PATEL, D. Giant left atrial: a case repport. Angiology, 42: 52 $54,1991$.

GROSS, L. - Lesion of the left auricle in rheumatic fever. Amer. J. Pathol., 11: 711, 1935.

54 GUEST, J. L.; HALL, D. P.; ELLISON, R. G. Symmetrical gangrene following insertion of ball valve mitral prosthesis in a patient with giant left atrium. $J$. Thorac. Cardiovasc. Surg., 49: 550, 1965.

GUIRAUDON, G. M.; CAMPBEL, C. S.; JONES, D. L. - Combined sinoatrial node ventricular node isolation; a new surgical alternative to His bundle ablation in patients with atrial fibrillation. Circulation, 72: 11-22, 1985.

56 HALL, J. \& DOBBS, R. H. - Cerebral emboli from aneurysm of left atrial appendage. Proc. Roy. Soc. Med., 62: 911-915, 1969.

57 HAMMERMAN, A. M. \& FISCHER, K. C. - The CT appearance of the giant left atrium. Clin. Imaging, 14: $305-308,1990$

58 HARMJANZ, K.; KOCHSIEK, A.; HEIMBURG, $P$. EMMERICH, J. - Die mitral insufizienz mit normal Drückhohe und normalen druckablauf im linken vorhof bei grossen regurgitationsvolumen. $Z$. Kreislauf Forsch, 55: 217, 1966

59 HEWETT, P. G. - Aneurysmal dilatation of left auricle with thickening and contraction of left auriculoventricular opening. Trans. Pathol. Soc. London, 2: 193, 1849/1850.

60 HIROSAWA, K.; SEKIGUSHI, M.; KASANUKI, L. Natural history of atrial fibrillation. Heart Vessels, 2: $14-23,1987$.

61 HORN, E. M.; DANILO, P.; APFELBAUM, M. A.; BARR M. L.; PEPINO, P.; POWERS, E. R.; SMITH, C. R.; ROSE, E. A.; BILEZIKIAN, J. P. - Beta-adrenergic receptor sensitivity and guanine nucleotide regulatory proteins in transplanted human hearts and autotransplanted baboons. Transplantation, 52: 960 966, 1991.

62 HOUGEN, T. J.; MULDER, D. G.; GYEPE, M. T. Aneurysm of the left atrium. Am. J. Cardiol., 33 $557-561,1974$

63 ISOMURA, T.; HISATOMI, K.; HIRANO, A.; MARUYAMA, H.; KOSUKA, K.; OHISHI, K. - Left atrial plication and mitral valve replacement for giant left atrium acompanying mitral lesion. J. Card. Surg., 8: $365-370,1993$.

64 JACKMAN, W. M.; FRIDAY, K. J.; ANDERSON, J. L.; ALIOT, E. M.; CLARK, M.; LAZZARA, R. - The long QT syndromes: a critical review, new clinical observations and unifying hypothesis. Prog. Cardiovasc. Dis., 31: 115-172, 1988.

65 JAZBIK, J. C.; COUTINHO, J. H.; AMAR, M. R.; SILVA S. R.; JAZBIK, J.; JAZBIK, A. T.; RODRIGUEZ, J.; PINTO, J. C.; GUEDES, M. V.; BONFIN, A. S.; 
BATISTA, R. J. V.; FRANZONI, M.; PRÉCOMA, D.; BOCHINO, L.; NERY, P.; OLIVEIRA, E.; CARVALHO, R.; TAKESHITA, N.; FURUKAWA, M.; THOME, L.; LINO, F. J.; SANTOS, J. L. V.; CUNHA, M. A. - Autotransplante cardíaco: um novo método no tratamento de problemas cardiacos complexos.. Rev. Bras. Cir. Cardiovasc., 10 (2): 90-100, 1995.

LOPES, A. M.; VALENCIA, A. V.; MEIER, M. A. JASBIK, W. - Tratamento cirúrgico da fibrilação atrial em pacientes com insuficiência mitral: proposta inicial de uma nova abordagem cirúrgica. Rev. SOCERJ, 6: 142-145, 1993.

JOHNSON, J.; DANIELSON, G. K.; MAC VAUGH, H. III; JOYNER, C. R. - Plication of the giant left atrium at operation for severe mitral regurgitation. Surgery, 61: 118-121, 1967.

KAWAZOE, K.; BEPPU, S.; TAKAHARA, Y.; NAKAJIMA, N.; TANAKA, K.; ICHIHASHI, K.; FUGITA, T.; MANABE, H. - Surgical treatment of giant left atrium combined with mitral valvular disease. J. Thorac. Cardiovasc. Surg., 85: 885-892, 1983.

KAYE, M. P.; RANDALL, W. C.; HAGEMAN, G. R.; GEISS, W. P. \& PRIOLA, D. V. - Chronology and mode of reinnervation of the surgically denervated canine heart: functional and chemical correlates. Am. J. Physiol., 233: 431-437, 1977.

KENT, E. M.; FISHER, D. L.; FORD, V.B.; NEVILLE, J. F. - Mitral valve surgery and left heart catheterization in giant left atrium. Arch. Surg., 73: 503-507, 1956.

KITAMURA, N.; YAMAGUSHI, A.; MIKI, T.; NOJI, S.; OTAKI, M.; KURATA, A. - Autotransplantation as optimal technique for recurrent malignant myxoma of left ventricle. Nippon Kyobu Geka Gakkai Zasshi, 41: 445-4511, 1993.

71 KNUDSEN, F. D.; DAILY, P. O.; STINSON, E. B. SHUMWAY, N. E. - Surgical treatment of the gigantic left atrium. Surg. Ginecol. Obstet., 555-570., 1969.

KONDO, Y.; MATHENY, J. L.; HARDY, J. D. - Autonomic reinnervation of cardiac transplants: further observations in dogs and rhesus monkeys. Ann. of Surg., 176: 42-48, 1972.

KONSTANTINOV, B. A.; CHEREPENIN, L. P.; TARICHKO, I. V.; RASULOV, I. R.; NECHAENKO, M. A. - Atrioplastika pri khirurgicheskoi korrektsii mitral 'nogo' poroka, oslozhnennogo levoi atriomegaliei. Grud. Serdechnososudistaia Khir., 2: 3-8, 1990. Responses to electrical and reflex autonomic stimulation in dogs with cardiac transplantation before and after reinnervation. J. Thorac. Cardiovasc. Surg., 59: 382-392, 1970

KONTOS, H. A.; ZUBAIR, H.; THAMES, M. D.; LOWER R. R. - Circulatory responses to hemorrhage in dogs with cardiac autotransplantation. Am. J. Physiol. 220: $621-623,1971$.

KORYTNIKOV, K. I. \& KORNEEV, N. V. - Differentsial 'naia ekhokardiogra ficheskaia diagnostika gigantskogo levogo predsesdiia i perikardial 'nogo vypotadifferential. Ter. Arkh., 59: 74-76, 1987.

KRONZON, I. \& MEHTA, S. - Giant left atrium. Chest, 65: $677-679,1974$.

KRUEGER, S. K.; FERLIK, R. M.; MOORING, P. K. Left atrial appendage aneurysm: correlation of noninvasive with clinical and surgical findings: report of a case. Circulation, 52: 732-738, 1975.
KRUSTINOV, G.; MILEV, M.; TOPALOV, I. - Autologous and homologous transplantation of the heart with both lungs in an experiment. Khirurgiia (Sofiia), 24: 444449,1971

80 LANG, T. W.; BAZIKA, V:; PAPPELBAUM, S.; GOULD, H.; BERNSTEIN, H.; HERROLD, G.; CORDAY, E. Autotransplanted heart-lung and cerebral venous shunt preparations: two new technics for pharmacologic assay of cardiovascular drugs. $A m$. J. Cardiol., 16: 695-700, 1965.

81 LEMIRE, F.; TAKIK, A. J.; HAGLER, D. J. - Asymetric left atrial enlargement:an echocardiographic observation. Chest, 69: 779-781, 1976.

82 LE ROUX, B. T. \& GOTSMAN, M. S. - Giant left atrium. Thorax, 25: 190-198, 1970.

LOWER, R. R.; STOFER, R. C.; SHUMWAY, N. E. Homovital transplantation of the heart. J. Thorac. Cardiovasc. Surg., 41: 196-204, 1961.

LUCAS, D. S.; MAHRER, P. R.; STEINBERG, I. Angiographic and physiological correlation in mitral stenosis. Circulation, 17: 567-575, 1958.

LUTEMBACHER, R. - Aneurysm of the left auricle. Arch. Mal. Coeur, 10: 145, 1917.

MCKENNEY, R. N. \& MELVIN, K. - Successful surgical reppair in a patient with mitral stenosis, calcified left atrium and severe tricuspid regurgitation with a giant right atrium. Can. J. Surg., 30: 264-265, 1987.

87 MCKEOWN, P. P.; TABAYASSHI, K.; MIYAMOTO, M THOMAS, R.; TOGO, T.; TSUBOI, H. - Cardiac transplantation without cardiopulmonary bypass: experimental model to study growth of the transplanted heart. Surgery, 104: 489-493, 1988.

MAHAIM, I. - Aneurysm of the left auricle. Medicine, 10 210,1929

MAHAPATA, S.; GOLBERG, M. J.; RYAN, J. M.; RUBENFIRE, M.; CASCADE, P. N. - Progressive extreme biatrial enlargement following mitral valve replacement. Chest, 84: 306-308, 1983.

90 MATSUDA, H.; NAKAO, M.; NOHARA, H.; HIGAMI, T. MUKOHARA, N.; ASADA, T.; OGAWA, K.; KAWAMURA, T. - The causes of prolonged postoperative respiratory care in mitral valve disease with a giant left atrium. Jpn. J. Thorac. Surg., 43: $172-182,1990$

MATSUDA, H.; NAKAO, M.; NOHARA, H.; HIGAMI, T.; MUKOHARA, N.; ASADA, T.; OGAWA, K.; KAWAMURA, T. - The causes of prolonged postoperative respiratory care in mitral valve disease with a giant left atrium. Kyobu Geka, 43: 172-177, 1990.

MINKOWSKI, O. - Demonstation eines herzens mit ungewohnlic starken dilation der vorhofe. Munchen Med. Wschr., 51: 182, 1904.

MOHANTY, P. K.; THAMES, M. D.; CAPEHEART, J. R. - Afferent reinnervation of the autotransplanted heart in dogs. J. Am. Coll. Cardiol., 7: 414, 1986.

94 MOSS, A. J.; SCHWARTZ, P. J.; CARAMPTON, R. S.; LOCATI, E.; CARLEEN, E. - The long QT syndrome: 
BATISTA, R. J. V.; FRANZONI, M.; PRÉCOMA, D.; BOCHINO, L.; NERY, P.; OLIVEIRA, E.; CARVALHO, R.; TAKESHITA, N.; FURUKAWA, M.; THOME, L.; LINO, F. J.; SANTOS, J. L. V.; CUNHA, M. A. - Autotransplante cardiaco: um novo método no tratamento de problemas cardíacos complexos.. Rev. Bras. Cir. Cardiovasc., 10 (2): 90-100, 1995.

a prospective international study. Circulation, 71 : 17-21, 1985.

95 NAKAE, S.; WEBB, W. R.; THEODORIDES, T.; SUGG, W. L. - Respiratory function following cardiopulmonary denervationin dog, cat, and monkey. Surg. Gynecol. Obstet., 125: 1285-1292, 1967.

96 NASSERI, M.; EISELE, R.; KOTTER, D.; KASPAR, F.; PAEPRER, H. - Ventilation and hemodynamics after complete cardiopulmonary autotransplantation. Langenbecks Arch. Chir. 331: 108-118, 1972.

97 NICHOLS, C. F. \& OSTRUM, H. W. - Unusual dilatation of the auricle. Am. Heart J., 8: 205-216, 1933.

98 NORVELL, J. E. \& LOWER, R. R. - Degeneration and regeneration of the nerves of the heart after transplantation. Transplantation, 15: 337-344, 1973.

99 NOVITSKY, D. COOPER, D. K.; ROSE, A. G.; ISAACS, S.; BONIASZCZUK, F.; SMITH, J. A.; REICHART, B.; ITURRALDE, M. P. - The effects of denervation and acute rejection of left ventricular volumes measured by radionuclide ventriculography following cardiac transplantation in the chacma baboon. Semin. Nucl. Med., 18: 213-220, 1988.

100 OWEN, I. \& FENTON, W. J. - A case of extreme dilatation of the left auricle of the heart. Trans. Clin. Soc. London, 34: 183-191, 1901.

101 PAGAN, V.; GRIGGIO, L.; LOSI, C.; CIGAINA, V.; ZOTTI, E. F. - Experimental autotransplant of the sinoatrial node in the area of the coronary sinus. Chir. Patol. Sper., 28: 73-79, 1980.

102 PARKER, J. O.; CONNELL, W. F.; LYNN, R. B. - Left atrial aneurysm. Am. J. Cardiol., 20: 579-582, 1967.

103 PARSONNET, A. E.; BERNSTEIN, A.; MARTLAND, H. S. - Massive left auricle with special reference to its etiology and mechanism: report of case. Am. Heart J., 31: 438-450, 1946.

104 PASTOR, B. H.; FORTE, A. L. - Idiopathic enlargement of the right atrium. AM. J. Cardiol., 8: 513-518, 1961.

105 PECH, H. J.; WARNKE, D.; MÜNTER, W. - Vergleich der berechneten mitralklappenoffnungsflasche mit dem angiokardiographisch ermittelten volumen des linken verhofes bei mitralstenosen. Fortsch. Roentgenstr., 108: 296, 1968.

106 PEEL, A. A. F. - Dilatation of the auricles resulting from heart block. Lancet, 2: 1248, 1929.

107 PEISS, C. N.; COOPER, T.; WILLMAN, V. L.; RANDALL, W. C. - Circulatory responses to electrical and reflex activation of the nervous system after cardiac denervation. Cir. Res., 19: 153-166, 1966.

108 PEPINO, P.; VOLPE, M.; ROSE, A.; PANZA, A.; LEMBO, G.; PIGNALOSA, S.; BARR, M. L.; COVINO, E.; CONDORELLI, M.; SMITH, C. R. - Effects of complete denervation on atrial natriuretic factor release in baboons. J. Surg. Res., 53: 43-47, 1992.

109 PFEIFFER, D.; FIEHRING, H.; WARNKE, H.; PECH, N. - Lechenie takhiaritmii u bolnoi s sindromon udlinennogo intervala Q-T s pomoshch'iu autotrans- plantasii sedtsa i stimuliatsii predserdii, upravliaemoi sinusovym uzlom. Kardiologiia, 30: 90-92, 1990.

110 PFEIFFER, D.; FIEHRING, H.; WARNKE, H.; PECH, H. J.; JENSSEN, S. - Treatment of tachyarrythmia in a patient with the long QT syndrome by autotransplantation of the heart and sinus nodetriggered atrial pacing. J. Thorac. Cardiovasc. Surg., 104: 491-494, 1992.

111 PICCOLI, G. P.; MASSINI, C.; EUSANIO, G.; BALLERINI, L.; IACOBONE, G.; SORO, A.; PALMINIELLO, A. - Giant left atrium and mitral valve disease: early and late results o surgical treatment in 40 cases. J. Thorac. Cardiovasc. Surg., 25: 328336,1984

112 PIWNIKA, A.; BLONDEAU, R.; D'ALLAINES, C.; DUBOST, C. - Le chirurgie des cardiopathies mitrales avec ectasie de l'oreillette gauche. Arch. Mal. Coeur, 60: 1532,1967

113 PLASCHKES, J.; BORMAN, J. B.; MERIN, G.; MILWIDSKY, H. - Giant left atrium in rheumatic heart disease. Ann. Surg., 174: 194-201, 1971.

114 REITZ, B. A.; BURTON, N. A.; JAMIESON, S. W. - Heart and lung transplantation, autotransplantation and allotransplantation in primates and extended survival. J. Thorac. Cardiovasc. Surg., 80:360, 1980.

115 REITZ, B. A.; DONG, E.; STINSON, E. B. - The Bainbridge reflex in canine cardiac auto transplants. Circulation, 43: 136, 1971

116 ROBERTS, W. C.; HUMPHRIES, J. O.; MORROW, A G. - Giant right atrium in rheumatic mitra stenosis: atrial enlargement restricted by mural calcification. A. Heart J., 79: 28-35, 1970.

117 ROGERS, W. R. \& WITTELS, B. - Extreme bilateral atriomegaly: review of the literature and repport of a case. Circulation, 15: 434-441, 1957.

118 ROSLER, H. \& WEISS, K. - Über die Veranderung des oesophagusverlaufes durch de vergrosserten linken vorhof. Fortschr. Geb. Roentgen Strahlen, 33: 717, 1925.

119 ROSSELET, A.; BACH, E. A. - Case of aneurysm of the left auricle. Arch. Mal. Coeur, 17: 145, 1924.

120 RUBIN, S. A. \& HIGHTOWER, C. W.; FLICKER, S. Giant right atrium after mitral valve replacement: plain film findings in 15 patients. Am. J. Roentegenol., 149: $257-260,1987$.

121 SAIGUSA, M.; MORIMOTO, K.; KOIKE, T.; HORI, T.; SATO, T. - Idiopathic enlargement of the right atrium. Jpn. Heart J., 3: 373-379, 1962.

122 SALERNO, T. A.; HOUCK, J. P.; BARROZO, C. A. M. - Retrograde continuous warm blood cardioplegia: a new concept in myocardial protection. Ann. Thorac. Surg., 51: 245-247, 1991.

123 SANDERUG, A.; GARMNA, D.; HATLE, L. - Aneurysmal dilatation of the left auricle. Scand. J. Thorac. Cardiovasc. Surg., 5: 143-146, 1971.

124 SCHELD, H. H.; NESTLE, H. W.; KLING, D.; STERMANN, N. A.; LANGEBARTELS, H.; HEHRLEIN, F. W. - Ressection of a tumor using 
BATISTA, R. J. V.; FRANZONI, M.; PRÉCOMA, D.; BOCHINO, L.; NERY, P.; OLIVEIRA, E.; CARVALHO, R.; TAKESHITA, N.; FURUKAWA, M.; THOME, L.; LINO, F. J.; SANTOS, J. L. V.; CUNHA, M. A. - Autotransplante cardiaco: um novo método no tratamento de problemas cardiacos complexos.. Rev. Bras. Cir. Cardiovasc., 10 (2): 90-100, 1995.

autotransplantation. J. Thorac. Cardiovasc. Surg., 36: $40-43,1988$.

125 SCHMIDT-HABELMAN, P.; PONTO, R.; ZAMORA, R.; CASTANEDA, A. - Pulmonary function studies following cardiopulmonary autotransolantation in primates (baboons) using Xenon-133. Res. Exp. Med. (Berlin), 157: 226-228, 1972.

$126 \mathrm{SCHOTT}$, A. - Extreme dilatation of the left auricle. Klin. Wchnschr., 3: 1067, 1924.

127 SCHWARTZ, S. I.; LILLEHEI, R. C.; SHIRES, G. T.; SPENCER, F. C.; STORER, E. - Principles of Surgery, vol. 2: 983, 1972.

128 SEKI, S.; TITUS, J. L.; DANIELSON, G. K. - Cardiac growth after cardiac autotransplantation in calves. J. Thorac. Cardiovasc. Surg., 62: 631-637, 1971.

129 SEMANS, J. H. \& TAUSSIG, H. B. - Congenital "aneurysmal" dilatation of the auricle. Bull. Johns Hopkins Hosp., 63: 404, 1938.

130 SERRA, A. J.; Mc NICHOLAS, K. W.; LEMOLE, G. M. - Giant left atrium as a cause of left pulmonary artery obstruction. Ann. Thorac Surg., 43: 329-331, 1987.

131 SHANAHAN, E. A.; DAGGETT, W. M.; LEAK, L. V. Functional, matabolic and structural studies of the chronic canine autotransplant. J. Surg. Res., 13: 269-281, 1972.

132 SHARER, R. M.; ANIX, W.; ALLEY, R. - Congenital enlargement of the left atrium. J. Thorac. Cardiovasc. Surg., 63: 292-299, 1972.

133 SHAW, H. B. - A case of extreme dilatation of the left auricle. Lancet, 2: 493-494, 1924.

134 SLOAN, S.; POLLOCK, R. C.; KIRSHBAUM, J.; FREEDMAN, T. - Massive dilatation of the left auricle: report of three cases. Ann. Intern. Med., 40: 75-91, 1954.

135 SMITH, N. T.; GERSHWIN, M. E.; HURLEY, E. J. Hemodynamic effects of ouabain on the surgically denervated, autotransplanted dog heart. Arch. Int. Pharmacodyn. Ther., 173: 95-114, 1968.

136 SMITH, N. T. \& HURLEY, E. J. - Citrate infusion in dogs following cardiac autotransplantation: studies on cardiovascular effects. Arch. Surg., 98: 44-48, 1969

137 STEEL, D. - Extreme dilatation of the left auricle. Am J. Roentgenol., 26: 67-73, 1931.

138 STEELE Jr., J. M. \& PATTERSON, R. - Distortion of the bronchi by left auricle enlargement. Am. Heart J., 4: $692,1928$.

139 TAJIK, A. J.; BROADBENT, J. C.; SHATTENBERG, T. T. - Idiopathic right atrial enlargement with pericardial effusion. Chest, 63: 450-453, 1973.

140 TAUSSIG, B. L. - A case of ticuspid stenosis with enormous dilatation of the right auricle. Am. Heart J., 14: 744-752, 1937.

141 TAYLOR, D. G. - Preservation and reimplantation of the heart in dogs. Ann. Chir. Thorac. Cardiovasc., 5: $247-248,1966$.
142 TENCKHOFF, L.; STAMM, S. J.; BECKWITH, J. B. Sudden death in idiopathic (congenital) right atrial enlargement. Circulation, 40: 227-235, 1969.

143 THADANI, V.; WHITAKER, W.; WATSON, D. A. Congenital intrapericardial aneurysm of the left atrium. Thorax, 30: 102-109, 1975.

144 THAMES, M. D.; ZUBAIR, H.; BRACKETT, N. C.; LOWER, R. R.; KONTOS, H. A. - Plasma renin responses to hemorrage after cardiac autotransplantation. $A m$. J. Physiol, 221: 1115-1119, 1971.

145 TILL, J. A.; SHINEBOURNE, E. A.; PEPPER, J.; CAMM, A. J.; WARD, D. E. - Complete denervation of the heart in a child with congenital long QT and deafness. Am. J. Cardiol., 62: 1319-1321, 1988.

146 TOUNTAS, C.; AREALIS, E.; ANDREADIS, P.; KIRIAKOU, K. - Total heart transplantation with preservation of its anatomo-physiologic relations: personal technique. J. Chir. (Paris), 97: 79-86, 1969.

147 TOUNTAS, C.; KIRIAKOU, K.; KARAPISTOLIS, E.; AREALIS, E.; GEORGOPOULOS, A.; ANDROULAKIS, G. - Heart autotransplantation in dogs: a new techical approach. Bull. Soc. Int. Chir., 28: 219-223, 1969.

148 VENNER, A. - Massive left atrium and mitral valvotomy. Brit. Med. J., 1: 1359, 1954.

149 WANG, T.; ANAGNOSTOPOULOS, C. E.; RESNEKOV, L. - Aneurysm of the body of the left atrium presenting with chest pain. Chest, 67: 226-229, 1975.

150 WILLIAMS, J. M.; UNGERLEIDER, R. M.; LOFLAND, G. K.; COX, J. L. - Left atrial isolation: new technique for the treatment of supraventricular arrythmias. $J$. Thorac. Cardiovasc. Surg., 80: 373-380, 1980.

151 WILLMAN, V. L.; COOPER, T.; CION, L. G. . Autotransplantation of the heart. Surg. Gynecol. Obstet., 115: 299, 1962.

152 WILLMAN, V. L.; COOPER, T.; HANLON, C. R. Coronary blood flow in dogs after cardiac transplantation. JAMA, 195: 206-207, 1966.

153 WILLMAN, V. L.; COOPER, T.; HANLON, C. R. - Return of neural responses after autotransplantation of the heart. Am. J. Physiol., 207: 187-189, 1964.

154 WILLMAN, V. L.; COOPER, T.; KAIZER, G. C.; HANLON, C. R. - Cardiovascular response after cardiac autotransplantation in primate. Arch. Surg., 91: 805$806,1965$.

155 WILLMAN, V. L.; MERJAVY, J. P.; PENNELL, R. HANLON, C. R. - Response of the autotransplanted heart to blood volume expansion. Ann. Surg., 166: $513,1967$.

156 WONG, C. K.; LAU, C, P.; CHENG, C. H.; LEUNG, W. H.; CHANG, T. Y.; PUN, K. K. - Elevated atrial natriuretic peptide level in giant atrium complicating Marfan's syndrome. Eur. Heart J., 10: 863-864, 1989.

157 ZIMIN, N. K.; KORMER, A. I. - Experimental autotransplantation of the heart. Kardiologiia, 17: 126-130, 1977. 